\title{
A nesprin-4/kinesin-1 cargo model for nucleus positioning in cochlear outer hair cells
}

\author{
Shahar Taiber ${ }^{1,2}$, Oren Gozlan², Roie Cohen², Leonarde Andrade ${ }^{3}$, Yehu Moran ${ }^{4}$, Rebecca Hipp ${ }^{5}$,
} Matthew W. Kelley ${ }^{5}$, Uri Manor ${ }^{3}$, David Sprinzak ${ }^{2 *}$, Karen B. Avraham ${ }^{1 *}$.

${ }^{1}$ Department of Human Molecular Genetics \& Biochemistry, Faculty of Medicine \& Sagol School of Neuroscience, Tel Aviv University, Tel Aviv 6997801, Israel; ${ }^{2}$ School of Neurobiology, Biochemistry and Biophysics, George S. Wise Faculty of Life Sciences, Tel Aviv University, Tel Aviv 6997801, Israel; ${ }^{3}$ Waitt Advanced Biophotonics Center, Salk Institute for Biological Studies, La Jolla, CA, USA; ${ }^{4}$ Department of Ecology, Evolution and Behavior, Alexander Silberman Institute of Life Sciences, Faculty of Science, The Hebrew University of Jerusalem, Jerusalem 9190401, Israel; ${ }^{5}$ Laboratory of Cochlear Development, National Institute on Deafness and Other Communication Disorders, NIH, Bethesda, Maryland, USA.

*Corresponding authors

David Sprinzak, davidsp@tauex.tau.ac.il

Karen B. Avraham, karena@tauex.tau.ac.il

ORCIDS

Shahar Taiber: 0000-0002-0787-4216

Yehu Moran: 0000-0001-9928-9294

Matthew W. Kelley: 0000-0001-7367-8697

Uri Manor: 0000-0002-9802-1955

David Sprinzak: 0000-0001-6776-6957

Karen B. Avraham: 0000-0002-4913-251X 


\begin{abstract}
Nuclear positioning is important for the functionality of many cell types and is mediated by interactions of cytoskeletal elements and nucleoskeleton proteins. Nesprin proteins, part of the linker of nucleoskeleton and cytoskeleton complex, have been shown to participate in nuclear positioning in multiple cell types. Outer hair cells (OHCs) in the inner ear are specialized sensory epithelial cells that utilize somatic electromotility to amplify auditory signals in the cochlea. Recently, nesprin-4 (encoded by Syne4) was shown to play a crucial role in nucleus positioning in OHCs. Syne4 deficiency in humans and mice leads to mislocalization of the $\mathrm{OHC}$ nuclei and cell death resulting in deafness. However, it is unknown how nesprin-4 mediates the position of the nucleus, and which other molecular components are involved in this process. Here, we show that the interaction of nesprin- 4 and the microtubule motor kinesin- 1 is mediated by a conserved 4 amino-acid motif. Using in-vivo AAV gene delivery, we show that this interaction is critical for nucleus positioning and hearing in mice. Nuclear mislocalization and cell death of OHCs coincide with the onset of hearing and electromotility and are solely restricted to outer, but not inner, hair cells. Overall, our results suggest that $\mathrm{OHCs}$ require unique cellular machinery for proper nucleus positioning at the onset of electromotility. This machinery relies on the interaction between nesprin-4 and kinesin-1 motors supporting a microtubule cargo model for nucleus positioning.
\end{abstract}

\title{
Introduction
}

Nuclear positioning and movement are integral to cell division, migration and function. Cells have evolved a plethora of mechanisms by which internal and external forces are transmitted to the nucleus, allowing its movement and positioning. The linker of nuceloskeleton and cytoskeleton (LINC) complex is a highly conserved complex of nuclear envelope proteins that has been implicated in nuclear positioning across species and cell types. The LINC complex consists of two families of proteins; SUN (Sad1, UNC-84) proteins that reside in the inner nuclear membrane (INM) and nesprin proteins that reside in the outer nuclear membrane (ONM) (Haque et al, 2006). 
In mammals, five members were identified in the nesprin family to date, nesprin-1, nesprin2, nesprin-3, nesprin-4 (Roux et al, 2009) (roux), and KASH5 (Grady et al, 2005; Wilhelmsen et al, 2005; Roux et al, 2009; Morimoto et al, 2012). All nesprin proteins are comprised of a C-terminus KASH domain and cytoplasmic spectrin repeats (SRs). The KASH domain has a short transmembrane sequence and the rest of it is found in the perinuclear space between the INM and ONM, where it interacts with SUN1 and SUN2 proteins that in turn interact with the nuclear lamina (Crisp et al, 2006). Importantly, localization of nesprins to the nuclear envelope (NE) is dependent on the KASH domain (Padmakumar et al, 2005).

Unlike nesprin-1 and nesprin-2, with their "giant" isoform of up to 1014 kDA, nesprin-4 is only $44 \mathrm{kDa}$, showing conservation of the C-terminus KASH domain but little resemblance of the cytoplasmatic region to nesprin-1 and nesprin-2. Interestingly, expression of Syne4 is very sparse, and nesprin-4 deficiency in humans and mice was associated with no phenotype other than deafness (Horn et al, 2013). In Syne4\%- mice, nuclei of cochlear outer hair cells are dislocated from their basal position, which leads to $\mathrm{OHC}$ death. It was also shown that nesprin-4 interacts with kinesin-1, yet the functional role of this interaction remains unclear (Roux et al, 2009). A 4-amino acid motif in nesprin-2 was recently identified as critical for binding of kinesin-1 this binding was shown to be critical for nucleus positioning in myotubes in a cargo model (Wilson \& Holzbaur, 2015). The authors were able to identify this motif in nesprin-4 as well and speculated that it would mediate the interaction of nesprin-4 with kinesin-1.

In the cochlea, expression of Syne4 is detected in the two types of mechanosensitive hair cells, inner hair cells (IHC) and outer hair cells (OHC) (Horn et al, 2013; Taiber et al, 2020). IHC receive $95 \%$ of the afferent cochlear innervation and are responsible for translating sound vibrations to neuronal signals (Raphael \& Altschuler, 2003). OHC have a unique role in amplifying basement membrane deflection by actively changing their length in response to changes in membrane potential, a behavior termed electromotility. By doing so, OHC contribute to the sensitivity and sharp frequency tuning of the auditory system (Mellado Lagarde et al, 2008). OHC are unique to mammals and unique molecular machinery has evolved to enable their specialized function (Ashmore, 2019). The discrepancy in the effect of Syne4 deficiency on IHC and OHC, suggest a role for Syne4 in electromotility. 
In this study we aimed at understanding whether $\mathrm{OHC}$ nucleus positioning relies on the interaction of nesprin- 4 with kinesin-1. We showed the interaction of nesprin- 4 and kinesin- 1 is mediated by the same 4 amino-acid motif in nesprin-4, as in nesprin-2, and that this interaction is essential for OHC survival. We used AAV gene delivery to test the role of nesprin-4/kinesin-1 interaction in-vivo and show that $\mathrm{OHC}$ nucleus positioning seems to be entirely dependent on this interaction. We further investigate the subcellular architecture of Syne ${ }^{\%} \mathrm{IHC}$ and $\mathrm{OHC}$ using electron microscopy and found that while nuclear architecture seems to be preserved, defects and in sub-membrane cisternae and autophagosomes were detected. Finally, examining the evolutionary development of nesprin-4 and the timeline of phenotype onset, suggests that the $\mathrm{OHC}$ phenotype observed in Syne4\%- mice could be triggered by electromotility.

\section{Results}

\section{A conserved motif mediates the interaction of nesprin-4 with kinesin motors}

Earlier work has identified a conserved 4 amino acid interaction domain, LEWD, in nesprins required for the interaction with kinesin-1 (Wilson \& Holzbaur, 2015). To test the hypothesis that the nucleus in $\mathrm{OHC}$ is positioned via kinesin-1 dependent cargo trafficking, we mutated the LEWD kinesin-binding domain in nesprin-4. We introduced a 2-amino acid substitution in this motif found at amino acids 243-246, LEWD to LEAA. Using co-immunoprecipitation in cell culture, we established that this mutation prevents the interaction of nesprin-4 and Kif5b (Figure 1A-B) showing that the LEWD is required for this interaction. To test the effect of mutating the LEWD interaction domain on the cellular distribution of nesprin-4, we transfected Chinese hamster ovary $(\mathrm{CHO})$ cells with plasmids encoding both versions of nesprin-4 fused to FLAG or an empty FLAG control (termed Nesp4 ${ }^{\mathrm{WT}}$ and Nesp4 ${ }^{\mathrm{AA}}$ ). We found that nesprin-4 ${ }^{\mathrm{AA}}$ localizes to the $\mathrm{NE}$ similarly to nesprin- $4^{\mathrm{WT}}$. Hence, nesprin- $4^{\mathrm{AA}}$ is properly translated and trafficked to the NE despite being unable to bind kinesin-1 (Figure 1D-E).

To study the effect of mutated nesprin4 in-vivo, we generated AAV vectors encoding either the wild type or the LEAA variant of nesprin-4 (termed AAV.Syne4 ${ }^{\mathrm{WT}}$ and AAV.Syne4 ${ }^{\mathrm{AA}}$, respectively). We used AAV9-PHP.B as we have shown previously that it can transduce virtually all IHC and OHC when injected during the first $48 \mathrm{~h}$ postnatally (Taiber et al, 2021). We have 
previously characterized the localization of exogenous AAV-expressed nesprin-4 (Taiber et al, 2021). Here, we injected mice with AAV.Syne $4^{A A}$ and saw that nesprin- $4^{A A}$ localizes to the NE of OHC, as we previously showed for WT nesprin-4, further indicating that it was translated and stable in-vivo (Supplementary Figure 1).

A

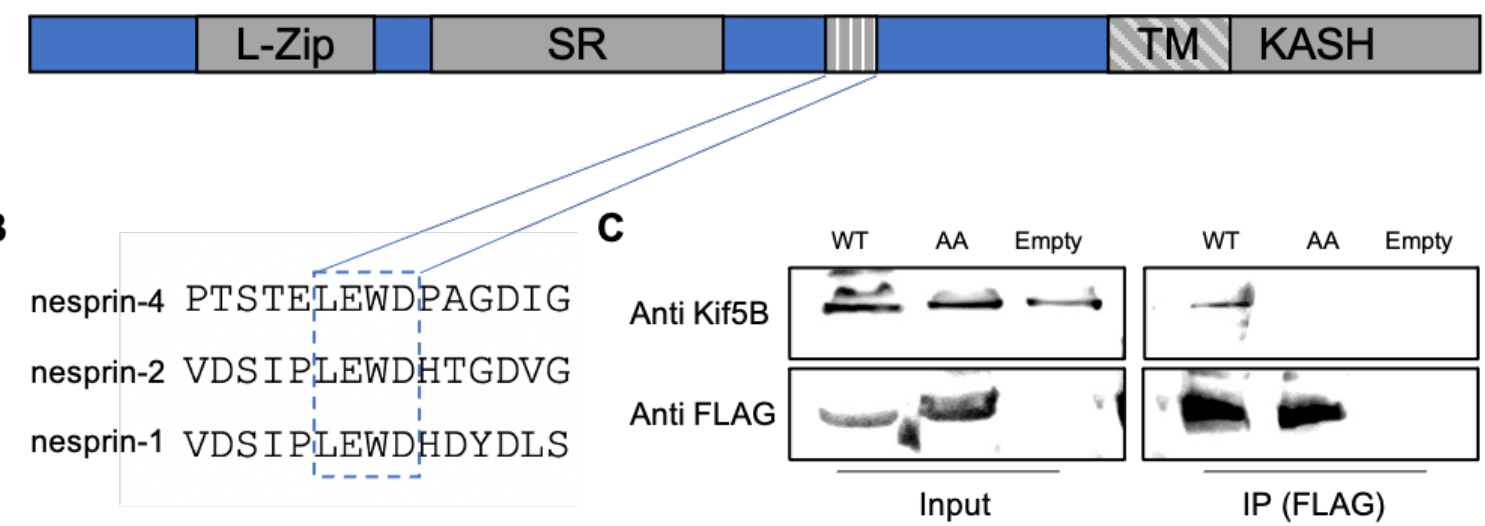

D
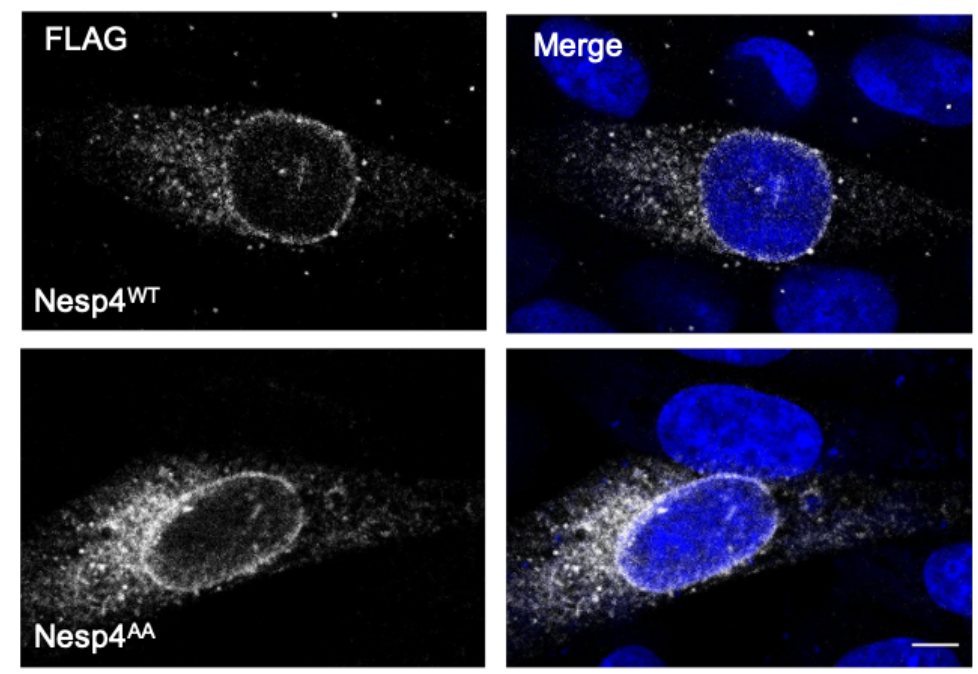

E

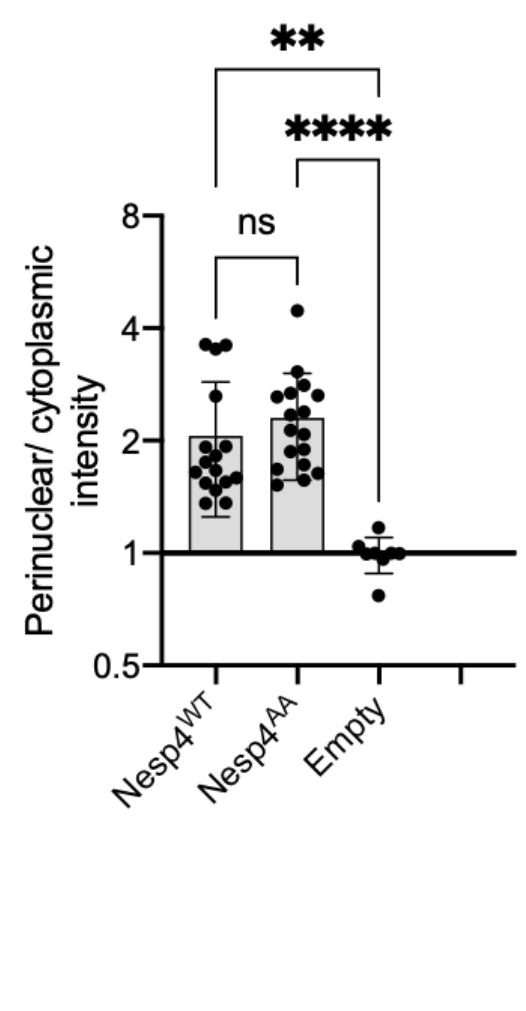

Figure 1. LEWD motif in nesprin-4 is required for interaction with kinesin-1. A. Schematic of nesprin-4 sequence showing the LEWD motif and other domains: TM = transmembrane, SR = spectrin repeat, L-Zip = leucine zipper. B. Alignment of human nesprin-4, nesprin-2, and nesprin-1 LEWD motifs. C. Coimmunopercipitation of LEWD and LEAA nesprin-4 with kif5b. Input = total lysate without precipitation. 
D. Nesprin- ${ }^{\mathrm{WT}}$ and nesprin- $4^{\mathrm{AA}}$ are recruited to the nuclear envelope. nesprin- 4 was labeled by FLAG (gray) and nuclei by DAPI (blue). E. Quantification of nuclear recruitment, $n=16$ cells for Nesp4 ${ }^{\mathrm{WT}}$, $\mathrm{n}=17$ cells for Nesp $4^{\mathrm{AA}}$ and $\mathrm{n}=8$ for empty FLAG, from three independent experiments. Plots show mean \pm SD Statistical test was Kruskal-Wallis test with Dunn's correction for multiple comparisons. ns=not significant, ${ }^{* * P}<0.01$, $* * * * P<0.0001$. Scale bar $=5 \mu \mathrm{m}$.

\section{Rescue of Syne 4 deafness is kinesin-1 dependent}

We have shown before that Syne $4^{-/}$mice show early-onset progressive deafness and that at P14 the nuclei of $\mathrm{OHC}$ are dislocated towards the apical surfaces, leading to rapid loss of OHC (Taiber et al, 2020). In addition, we have shown that a single injection of AAV9-PHP.B encoding Syne4 was sufficient to entirely prevent nuclear mislocation, $\mathrm{OHC}$ loss, and deafness in Syne ${ }^{-/}$mice. To test whether nesprin-4 function is indeed dependent on the interaction with kinesin-1, we injected Syne ${ }^{-/-}$mice with either AAV.Syne ${ }^{\mathrm{WT}}$ or AAV.Syne4 ${ }^{\mathrm{AA}}$ at P0-P1.5.

We observed that while AAV.Syne ${ }^{\mathrm{WT}}{ }^{\mathrm{T}}$ prevents nucleus mislocation in OHC, AAV.Syne ${ }^{\mathrm{AA}}$ fails to do so (Figure $2 A-B$ ). Moreover, unlike AAV.Syne ${ }^{\text {WT }}$, AAV.Syne4 ${ }^{\mathrm{AA}}$ does not improve OHC survival at $4 \mathrm{w}$ (Figure 2C-D). In contrast to $\mathrm{OHC}$, the position of IHC nuclei is not affected by the mutation or by treatment. Auditory brainstem response (ABR) of mice injected with AAV.Syne4 ${ }^{\text {WT }}$ is markedly improved at $4 \mathrm{w}$ with some treated Syne ${ }^{-/-}$showing thresholds indistinguishable from WT (Figure 2E). However, Syne $4^{\%}$ mice injected AAV.Syne4 ${ }^{\text {AA }}$ were not significantly different from untreated Syne4/- mice. Distortion-product otoacoustic emissions (DPOAE) are caused by the OHC electromotility-dependent cochlear amplifier, and thus we measured DPOAEs as an assay to assess $\mathrm{OHC}$ functionality in-vivo. In line with the ABR analysis, AAV.Syne ${ }^{\mathrm{AA}}$ did not improve DPOAE thresholds, while AAV.Syne4 ${ }^{\text {WT }}$ rescued thresholds in Syne4/- mice to WT levels (Figure 2F). Thus, the LEWD interaction domain in nesprin-4 is required for proper nuclear positioning and survival of $\mathrm{OHC}$. 
A

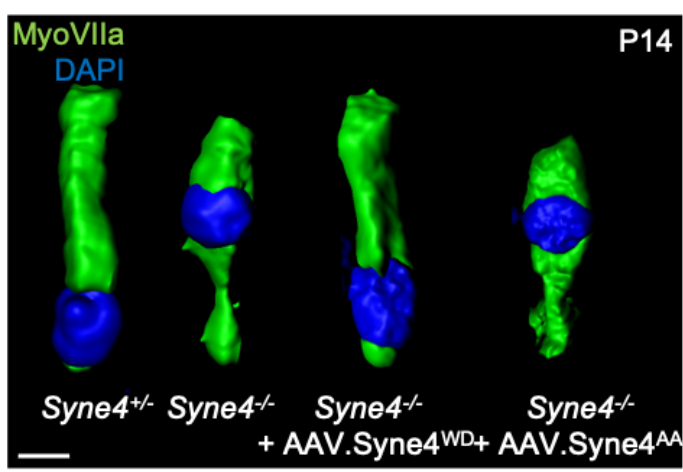

C

Syne $4^{+}$
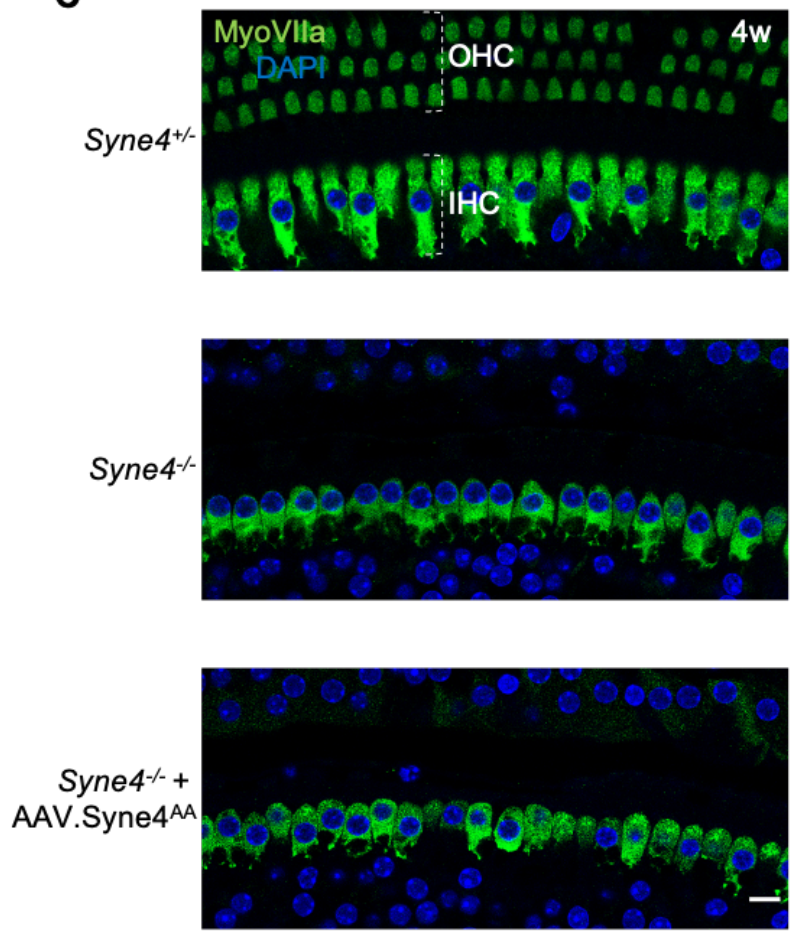

E

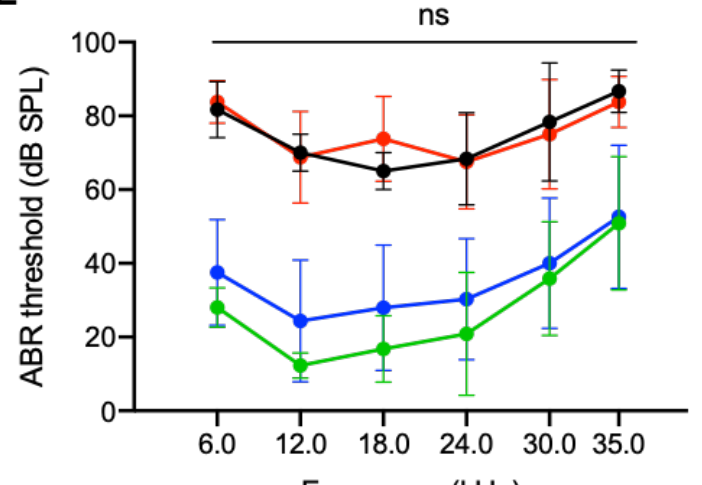
Frequency $(\mathrm{kHz})$
B
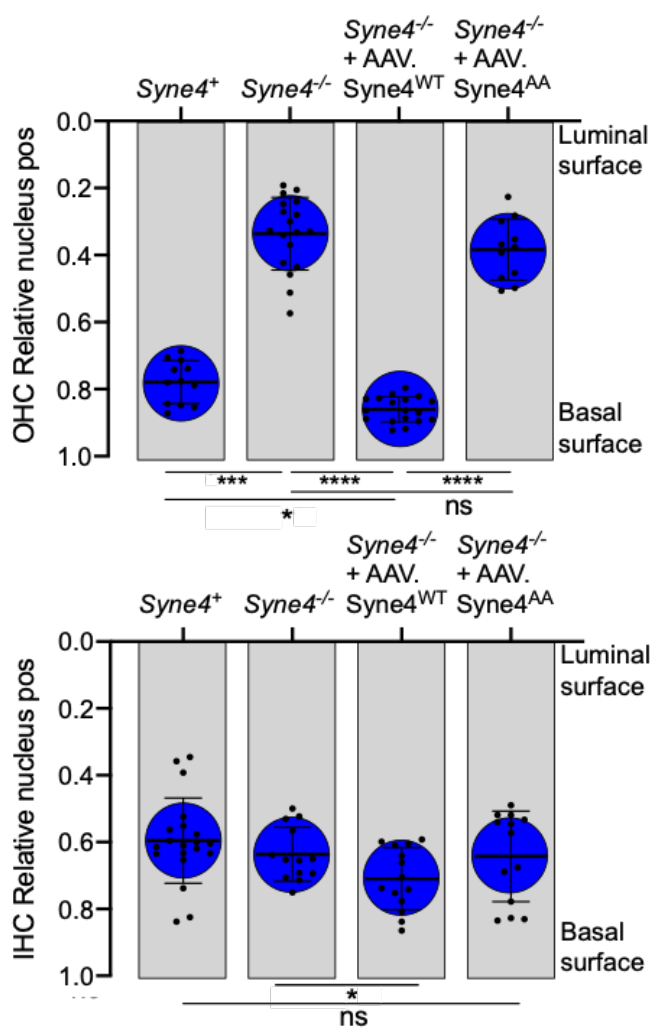

D
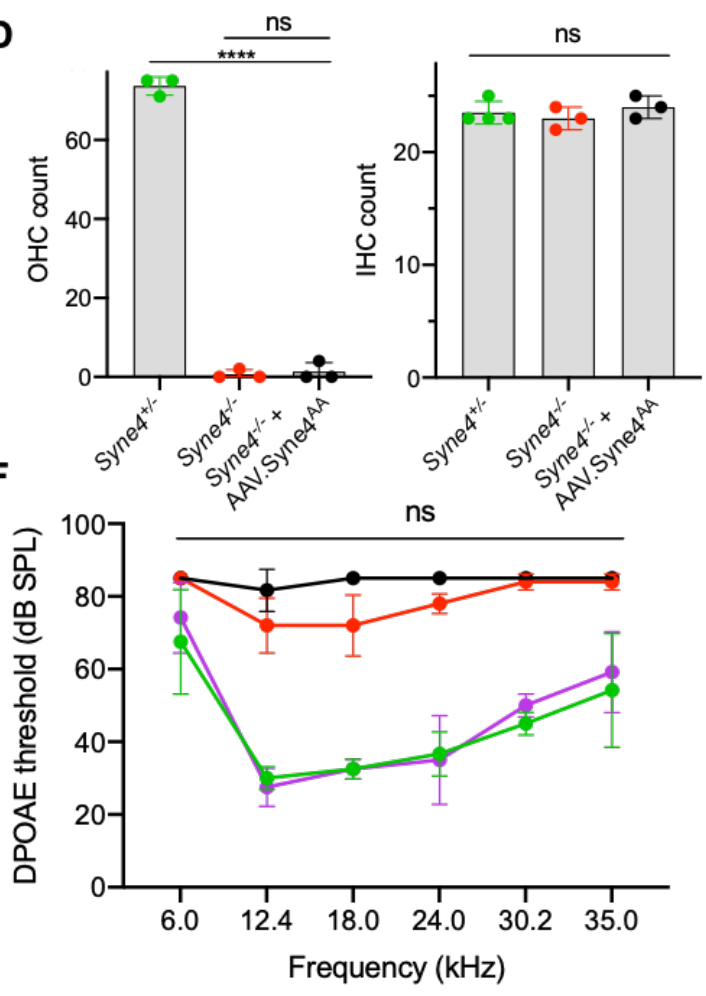

Syne $4^{-\%}+$ AAV.Syne $4^{\text {AA }}$ 
Figure 2. Mutation in kinesin-1 interaction motif abolishes the activity of nesprin-4 in the inner ear. A. 3D surface projection of P14 OHC from the $12 \mathrm{kHz}$ region of Syne $4^{+/+}$, Syne $4^{-/}$, Syne ${ }^{\%}$ injected with AAV.Syne $4^{\mathrm{WT}}$, and Syne $4^{-/}$injected with AAV.Syne $4^{\mathrm{AA}}$ mice. B. Image analysis quantification of relative nucleus position at $\mathrm{P} 14$ for $\mathrm{OHC}$ and IHC. A total of $12 \mathrm{OHC}$ and $20 \mathrm{IHC}$ from 2 Syne $4^{+/-}$mice, $18 \mathrm{OHC}$ and $13 \mathrm{IHC}$ from 3 Syne $4^{-}$mice, $18 \mathrm{OHC}$ and $14 \mathrm{IHC}$ from 3 Syne $4^{\%}$ mice injected with AAV.Syne $4^{\mathrm{WT}}$, and 11 $\mathrm{OHC}$ and $13 \mathrm{IHC}$ from $2 \mathrm{Syne}^{\%}$ mice injected with AAV.Syne4 ${ }^{\mathrm{AA}}$ were analyzed. C. Whole-mount immunofluorescence of the $12 \mathrm{kHz}$ region from $4 \mathrm{w}$ old Syne $4^{+/}$, Syne $4^{-/}$, and Syne $4^{-/}$mice injected with AAV.Syne $4^{\mathrm{AA}}$. Hair cells labeled by MyoVIla (green) and nuclei by DAPI (blue). D. OHC and IHC counts at the $12 \mathrm{kHz}$ region per $200 \mu \mathrm{m}$. E. ABR thresholds of $4 \mathrm{w}$ old Syne $4^{+/+}$, Syne $4^{-/}$, Syne $4^{-/}$injected with AAV.Syne4WT, and Syne $4^{-/}$injected with AAV.Syne4AA mice, $n=11$ for Syne $4^{+/+}, n=8$ for Syne $4^{-/}, n=20$ for Syne $4^{--}+$AAV.Syne ${ }^{\mathrm{WT}}, \mathrm{n}=3$ for Syne $4^{\%}+$ AAV.Syne $4^{\mathrm{AA}}$. F. DPOAE thresholds at $4 \mathrm{w}, \mathrm{n}=6$ for Syne $4^{+/+}, \mathrm{n}=5$ for Syne $4^{\%}, \mathrm{n}=6$ for Syne $4^{\%}+$ AAV.Syne $4^{\mathrm{WT}}, \mathrm{n}=3$ for Syne $4^{-}+$AAV.Syne $4^{\mathrm{AA}}$. Plots show mean \pm SD. Statistical tests were Kruskal-Wallis with Dunn's correction for multiple comparisons for B, one-way ANOVA for $D$ and 2-way ANOVA for $E$ and $F$ with Tukey's correction for multiple comparisons. ns=not significant, $* \mathrm{P}<0.05, * * * \mathrm{P}<0.001, * * * * \mathrm{P}<0.0001$. Scale bars $=10 \mu \mathrm{m}$.

\section{Nesprin- $4^{\mathrm{AA}}$ acts as a dominant regulator of nesprin-4 activity}

To verify that the inability of nesprin- $4^{\mathrm{AA}}$ to rescue $\mathrm{OHC}$ loss and hearing is directly due to the abolishment of interaction with kinesin-1, we tested whether overexpression of nesprin- $4^{\mathrm{AA}}$ will recapitulate the knockout phenotype by competing with the endogenous nesprin-4 over binding to SUN1. To this end, we injected Syne $4^{+/-}$mice (that have no phenotype) with either version of nesprin-4. While overexpression of nesprin- ${ }^{\mathrm{WT}}$ had no observable effect on $\mathrm{OHC}$ nuclear position at P14 or OHC survival at 4w, nesprin-4 ${ }^{\mathrm{AA}}$ leads to mislocation of $\mathrm{OHC}$ nuclei and $\mathrm{OHC}$ loss (Figure $3 A-D)$. Consistent with our previous observation, IHC nucleus position and IHC survival were not affected (Figure 3B-D). ABR results of Syne $4^{+/-}$mice injected with AAV.Syne4 ${ }^{\mathrm{AA}}$ showed only moderate levels of hearing loss, probably because only one ear was injected (Figure 3E). ABR electrodes were positioned to measure the injected ear, but it is known that there is contribution of both ears to the measured signal. DPOAE results measured from the injected ear showed a much clearer result, as massive loss of $\mathrm{OHC}$ in Syne ${ }^{+/-}$mice injected with AAV.Syne $4^{\mathrm{AA}}$ abolished cochlear amplification (Figure 3F). 
A
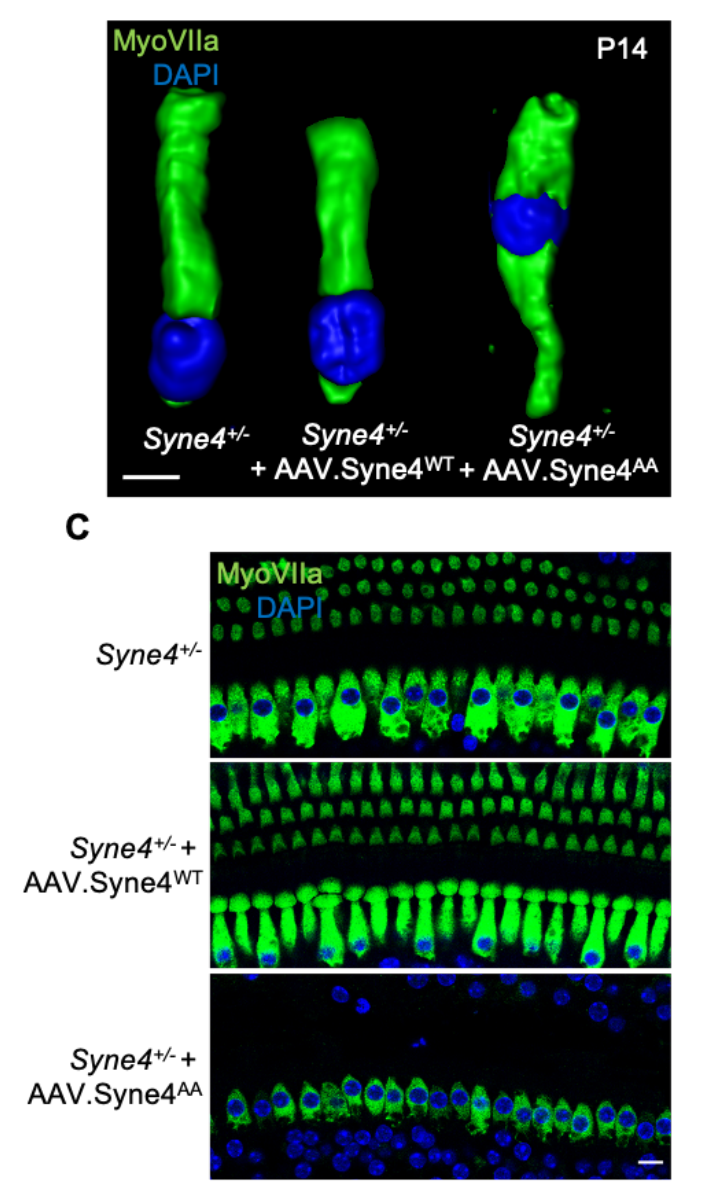

D
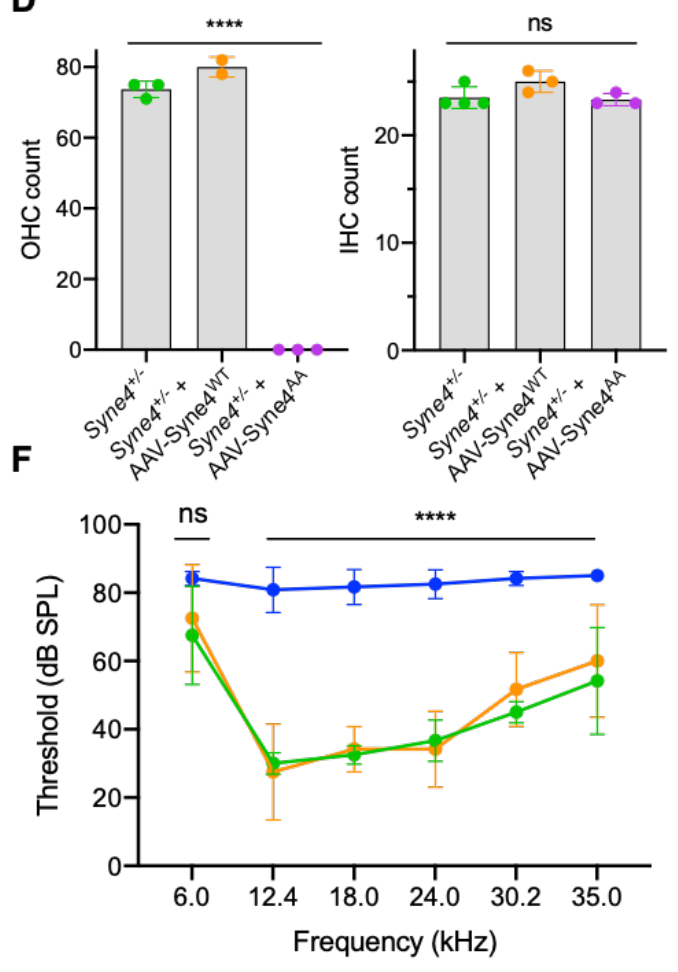

B
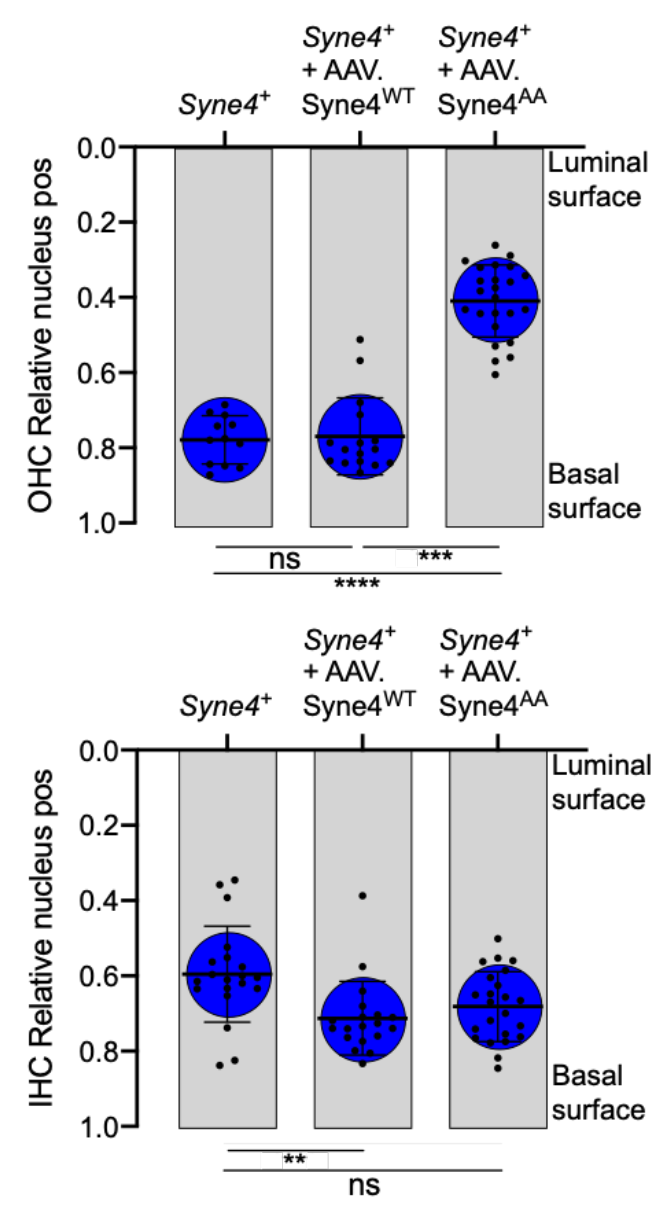

E

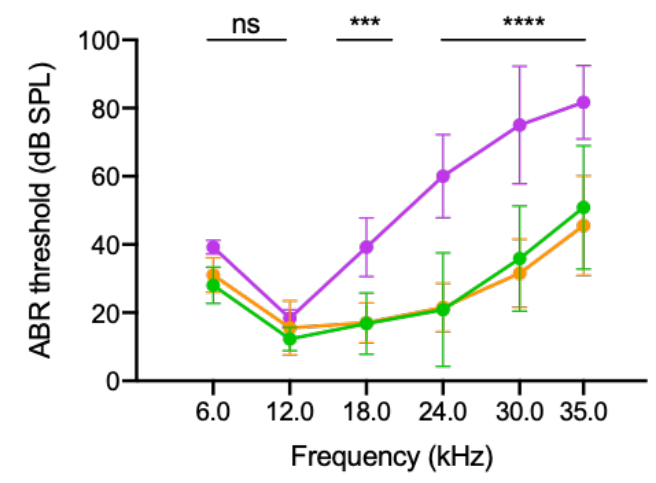

$\rightarrow$ Syne $4^{+}$

$\rightarrow$ Syne $4^{+}+$AAV.Syne $4^{\text {WT }}$

$\rightarrow$ Syne $4^{+}+$AAV.Syne $4^{A A}$ 
Figure 3. Overexpression of mutant nesprin- $4^{\mathrm{AA}}$ has a dominant negative effect on OHC. A. 3D surface projection of P14 OHC from the $12 \mathrm{kHz}$ region of Syne $4^{+/-}$, Syne $4^{+/-}$injected with AAV.Syne $4^{\mathrm{WT}}$, and Syne $4^{+/-}$ injected with AAV.Syne $4^{\mathrm{AA}}$ mice. B. Image analysis quantification of relative nucleus position at P14 for $\mathrm{OHC}$ and IHC. A total of $12 \mathrm{OHC}$ and $20 \mathrm{IHC}$ from 2 Syne $^{+/-}$mice, $16 \mathrm{OHC}$ and $19 \mathrm{IHC}$ from 4 Syne $4^{+/-}$mice injected with AAV.Syne $4^{\mathrm{WT}}$, and $24 \mathrm{OHC}$ and $23 \mathrm{IHC}$ from 4 Syne $4^{+/-}$mice injected with AAV.Syne $4^{\mathrm{AA}}$ were measured. C. Whole-mount immunofluorescence of the $12 \mathrm{kHz}$ region from $4 \mathrm{w}$ old Syne $4^{+/-}$and Syne $4^{+/-}$ injected with AAV.Syne $4^{\mathrm{AA}}$ mice. Hair cells labeled by MyoVIla (green) and nuclei by DAPI (blue). D. OHC and IHC counts at the $12 \mathrm{kHz}$ region per $200 \mathrm{um}$. E. ABR thresholds of $4 \mathrm{w}$ old Syne $4^{+/+}$, Syne $4^{+/}$injected

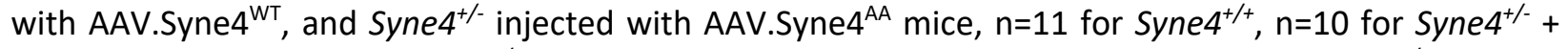
AAV.Syne $4^{\mathrm{WT}}$, and $\mathrm{n}=6$ for Syne $4^{+/-}+$AAV.Syne $4^{\mathrm{AA}}$. F. DPOAE thresholds at $4 \mathrm{w}, \mathrm{n}=6$ for Syne $4^{+/+}, \mathrm{n}=6$ for Syne $^{+/-}+$AAV.Syne $4^{\mathrm{WT}}$, and $\mathrm{n}=6$ for Syne $4^{+/}+$AAV.Syne ${ }^{\mathrm{AA}}$. Plots show mean \pm SD. Statistical tests were Kruskal-Wallis with Dunn's correction for multiple comparisons for B, one-way ANOVA for $D$ and 2-way ANOVA for $\mathrm{E}$ and $\mathrm{F}$ with Tukey's correction for multiple comparisons. ns=not significant, $* * \mathrm{P}<0.01$, $* * * \mathrm{P}>0.001, * * * * \mathrm{P}<0.0001$. Scale bars $=10 \mu \mathrm{m}$.

\section{Syne $\%$ OHC phenotype coincides with the onset of electromotility and hearing}

To understand why Syne4 deficiency leads to $\mathrm{OHC}$ death we investigated the temporal dynamics of Syne4 expression with respect to the onset of the phenotype. Single molecule fluorescent in situ hybridization (smFISH) performed at E16, P1 and P11 shows that the Syne4 is expressed by both $\mathrm{IHC}$ and $\mathrm{OHC}$ at early stages of development, in agreement with previous analyses we performed on transcriptome data (Figure 4A) (Taiber et al, 2021). We then analyzed the position of the nucleus in Syne ${ }^{-/}$mice at different ages and observed that while Syne4 is expressed early in embryonic development, nuclei are mislocated in Syne4\%- OHC only at P12-P14, coinciding with the onset of $\mathrm{OHC}$ electromotility and hearing (Figure 4B) (Bai et al, 2019): at P8 there is no difference in nucleus position between Syne $4^{--}$and Syne $4^{+} \mathrm{OHC}$ and by P12 the nuclei of Syne $4^{-}$ /- OHC is significantly mislocalized.

\section{Cellular defects in Syne4\% ${ }^{-/-}$OHC}

To study the ultrastructural effects of nucleus mislocation in Syne4\% OHC we performed TEM on P12 Syne $4^{+/+}$and Syne $4^{-/}$ears. We found the sub-membrane cisternae of Syne $4^{-/}$OHCs were compromised and autophagosomes were detected in their cytoplasm. These findings suggest cellular stress and damage due to loss of nesprin-4. Interestingly, we observed no obvious differences in the inner architecture of the nucleus (Figure $4 \mathrm{C}$ ). In both Syne $4^{-/-}$and Syne $4^{+/+} \mathrm{OHC}$ nuclei the NE was continuous, with no blebs or ruptures, and heterochromatin was similarly 
bioRxiv preprint doi: https://doi.org/10.1101/2021.10.10.463824; this version posted October 15, 2021. The copyright holder for this preprint (which was not certified by peer review) is the author/funder. All rights reserved. No reuse allowed without permission.

localized to the nuclear periphery. Overall, we did not observe any significant defects in subnuclear architecture, but did observe significant changes to other membrane components of the cell. 
bioRxiv preprint doi: https://doi.org/10.1101/2021.10.10.463824; this version posted October 15, 2021. The copyright holder for this preprint (which was not certified by peer review) is the author/funder. All rights reserved. No reuse allowed without permission.

A
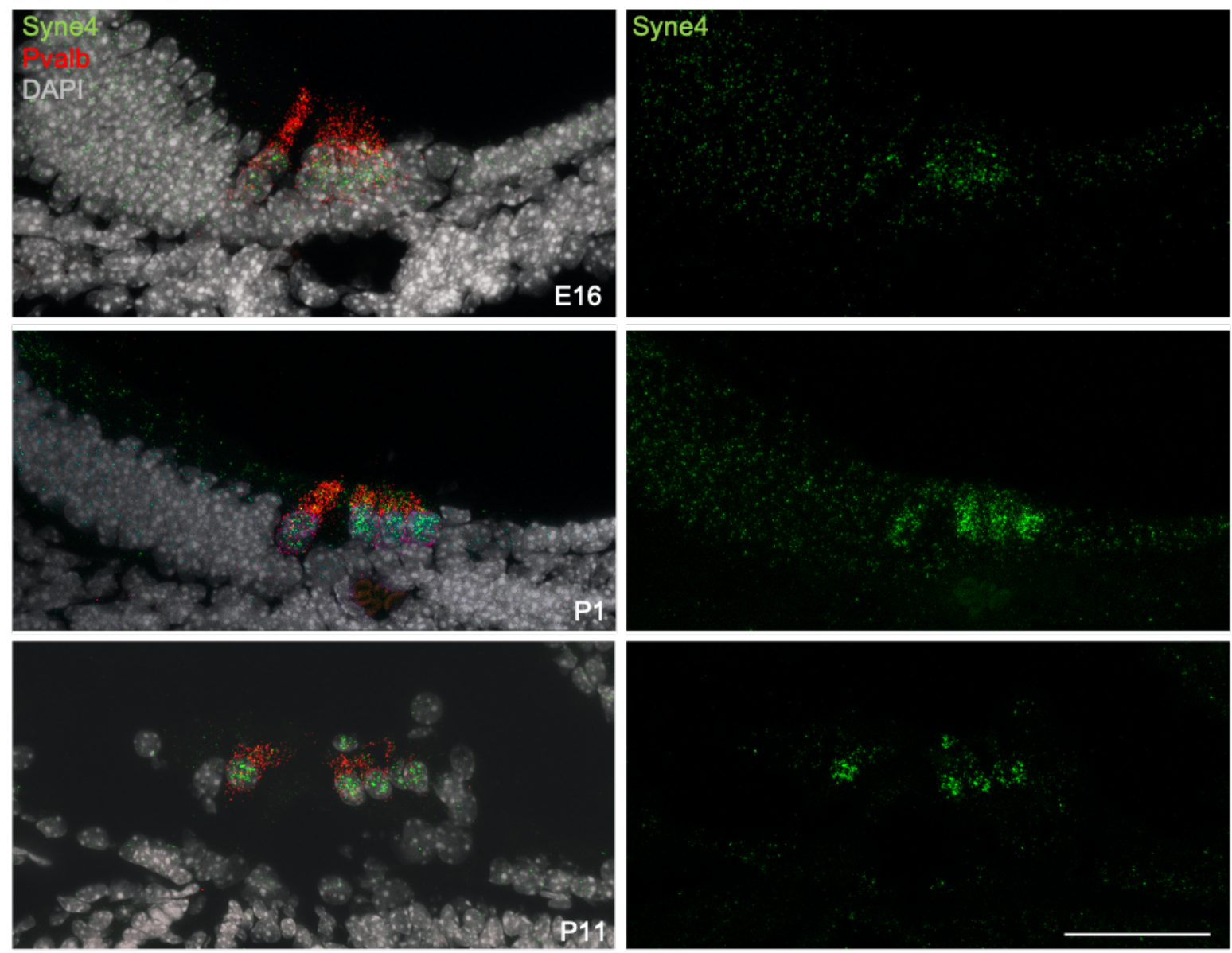

B

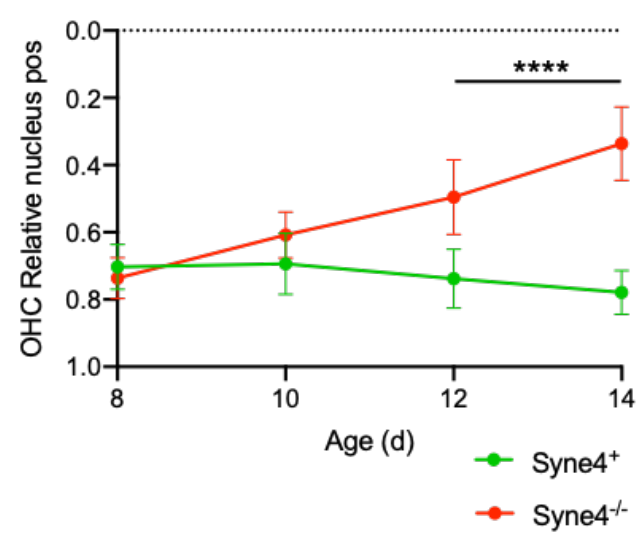

C

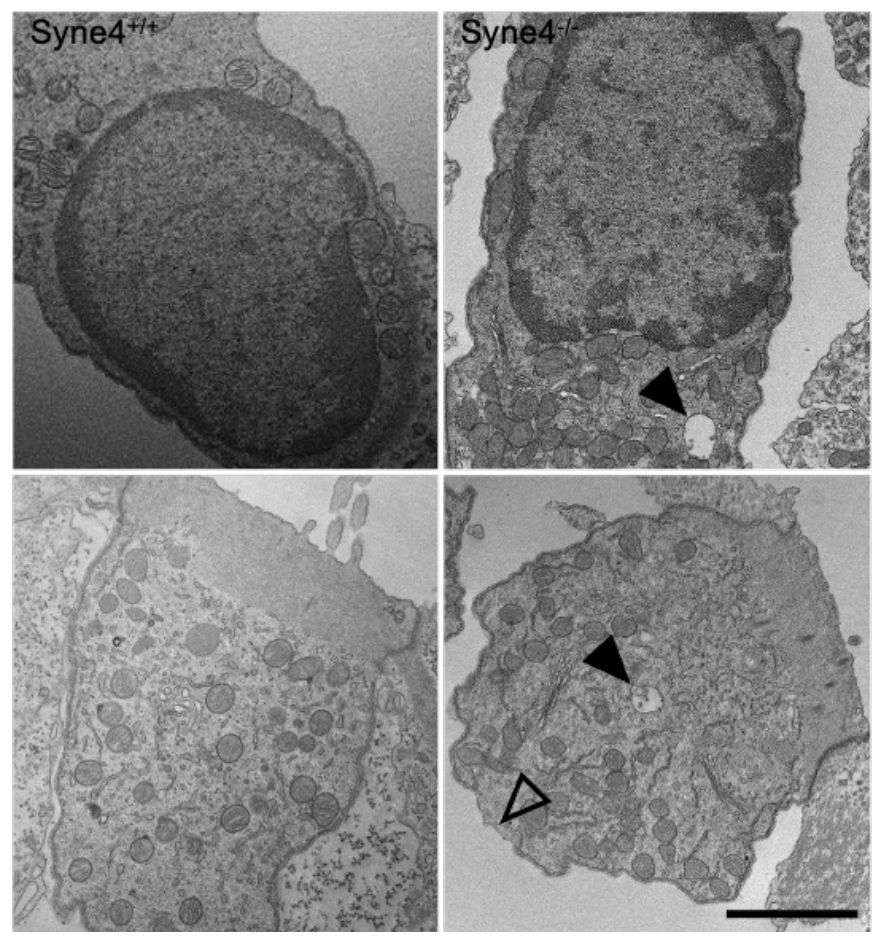


Figure 4. Syne $4^{-/}$phenotype is correlated to onset of electromotility and hearing. A. smFISH for Syne4 (green) and Pvalb (red) performed at E16, P1, and P11. Nuclei stained with DAPI (blue). B. Image analysis quantification of relative nucleus position at P8-P14 for OHC in Syne $4^{-/}$and Syne $4^{+}$mice. A total of $39 \mathrm{OHC}$ from 8 Syne $4^{+}$mice, and $64 \mathrm{OHC}$ from 11 Syne ${ }^{-/-}$mice were measured. C. TEM of P11 Syne $4^{-/}$and Syne $4^{+/+}$ mice. Open arrows denote sub-membrane cisternae defects, black arrows denote autophagosomes. Experiment was repeated 3 times. Plot shows mean \pm SD. Statistical test was multiple t-test with twostage step-up correction for multiple comparisons. ${ }^{* * * * P} \mathrm{P}<0.0001$. Scale bars $=50 \mu \mathrm{m}$ for $\mathrm{A}, 2 \mu \mathrm{m}$ for $\mathrm{C}$.

\section{Evolution of nesprin-4}

$\mathrm{OHC}$ are unique to mammals and have specialized proteins and structures that support their electromotility function. Considering the time of onset of nuclear dislocation, and the specificity to $\mathrm{OHC}$, we analyzed the evolution of the nesprin protein family in order to understand how nesprin-4 emerged. Sequences of Homo sapiens and Mus musculus nesprin proteins were aligned against homologous sequences from chicken (Gallus gallus), zebrafish (Danio rerio), and frog (Xenopus tropicalis). The results were used to build a phylogenetic tree of nesprin proteins (Figure 5). Nesprin-4 from Homo sapiens and Mus musculus, and Xenopus tropicalis cluster with a Danio rerio protein named "uncharacterized protein LOC777613". Inspection of the expression of this protein in published datasets via gEAR shows enrichment in HC (Orvis et al, 2021). Alignment of its sequence with the Homo sapiens and Mus musculus nesprin-4 paralogs suggest that this protein is in fact the zebrafish homolog of nesprin-4 (Figure 6). No hit was detected for nesprin4 or the KASH domain of nesprin-4 in the chicken genome. We then aligned Homo sapiens and Mus musculus nesprin proteins against their homologs from lancelet (Branchiostoma floridae), representing a non-vertebrate chordate branch that is relatively close to vertebrates, and observed that while nesprin-4 fails to align with any protein, nesprin-1 and nesprin-2 align with multiple proteins of the Branchiostoma nesprin family (Supplementary Text 1-3). Together, these results suggest that nesprin-4 could have evolved in the last common ancestor of vertebrates as a $\mathrm{HC}$ specific nesprin due to a truncation event in the other ancestral nesprin proteins. 


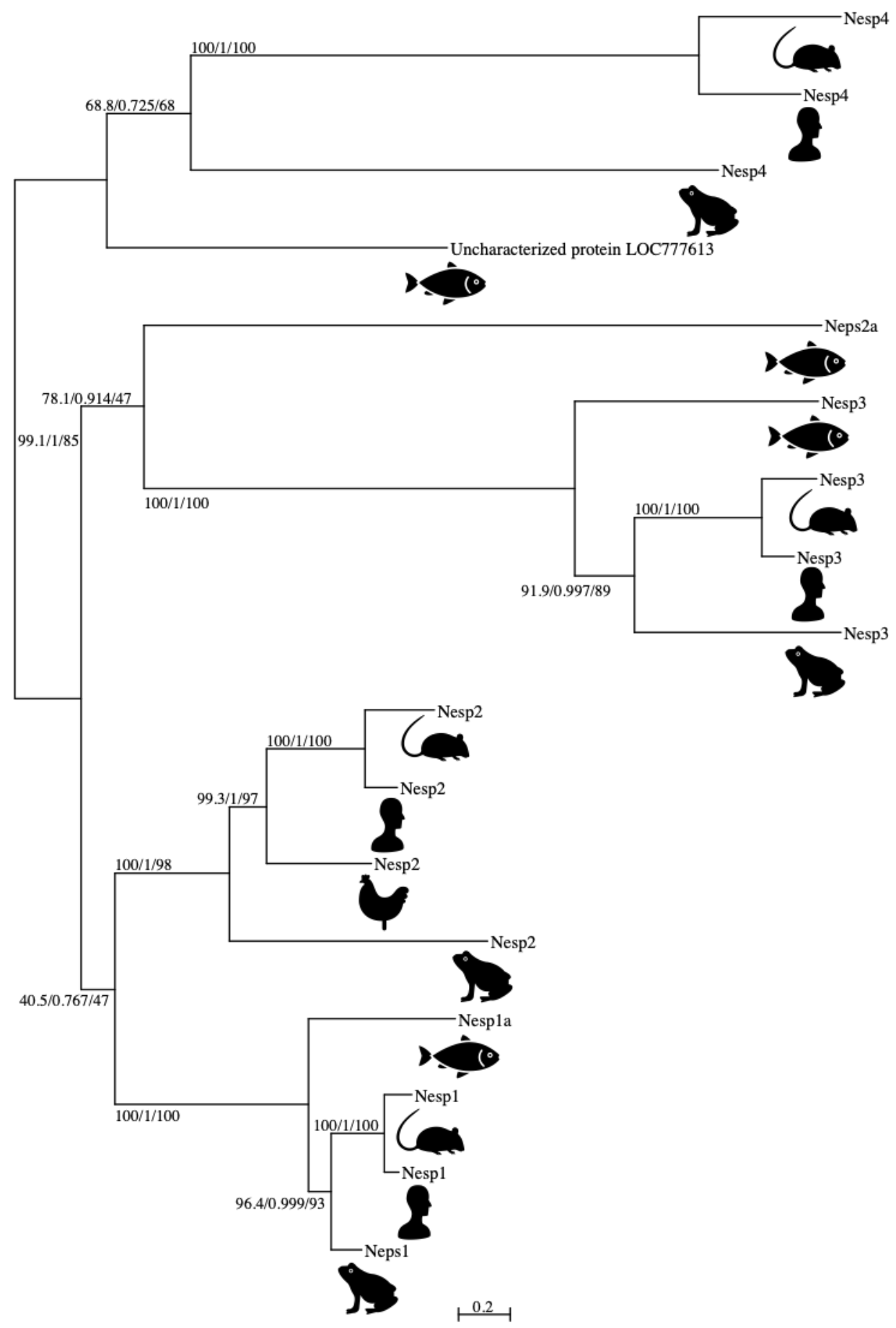

Figure 5. Phylogenetic analysis of nesprin proteins shows that nesprin-4 is conserved in vertebrates but is absent in invertebrates. Humanoid = Homo sapiens, rodent = Mus musculus, frog = Xenopus tropicalis, fish = Danio rerio, chicken = Gallus gallus. Units denote substitutions per site. Numbers next to each branch represent SH-Like, approximate Bayesian and ultrafast bootstrap support values, respectively. 
A

0

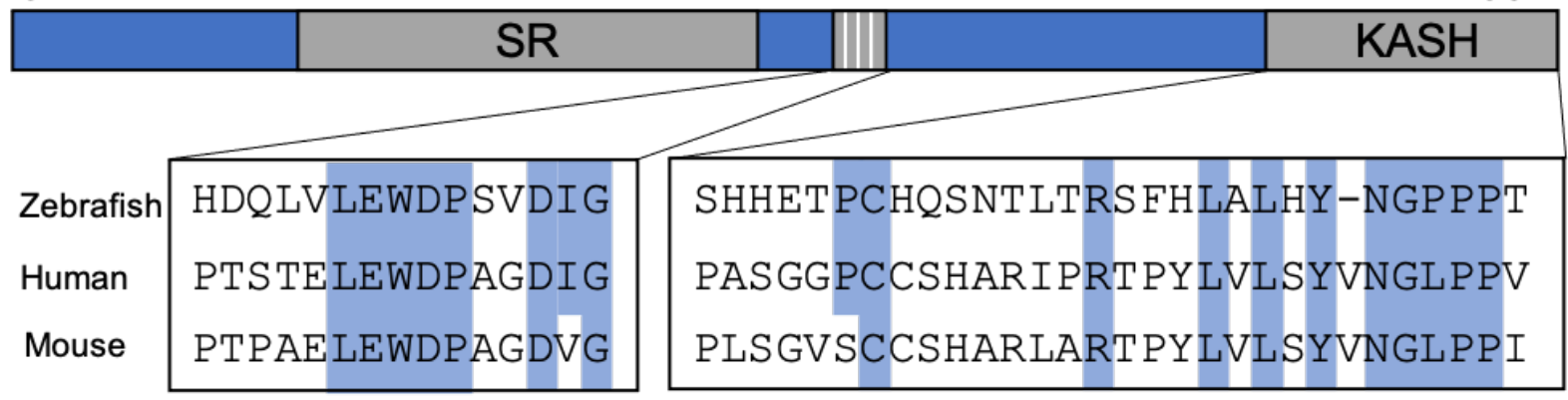

B

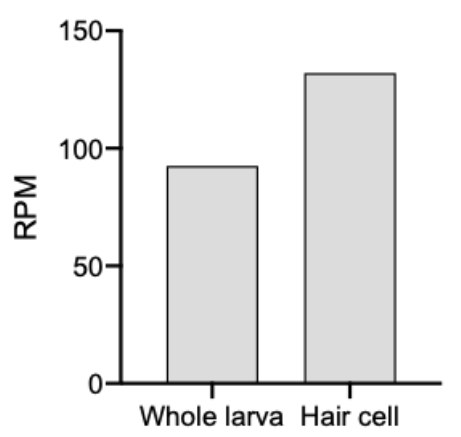

C

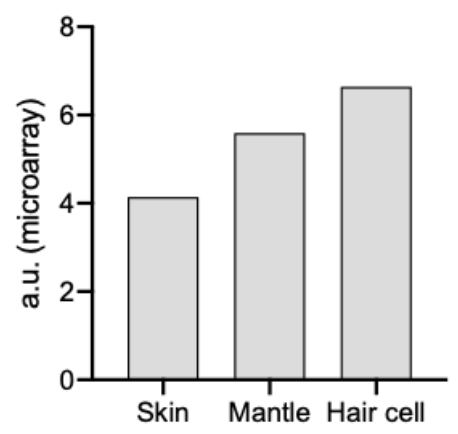

Figure 6. Identification of a new putative zebrafish nesprin-4. A. Alignment of the putative zebrafish nesprin-4 protein with human and mouse nesprin-4 proteins. The nuclear KASH domain and cytoplasmic kinesin-1 binding motif are highlighted. SR = spectrin repeat. B. Expression of putative zebrafish nesprin4 by RiboTag RNAseq (Matern et al, 2018). Data curated using gEAR (Orvis et al, 2021). C. Expression of putative zebrafish nesprin-4 by microarray (Steiner et al, 2014).

\section{Discussion}

Here, we provide evidence supporting a cargo model of nuclear positioning in $\mathrm{OHC}$, dependent on the interaction between nesprin-4 and kinesin-1. We used co-immunoprecipitation to show that nesprin-4 interacts with kinesin-1 light-chains through a conserved 4 amino acid motif, LEWD. Using in-vivo AAV gene delivery, we demonstrate that disruption of this motif creates an inactive nesprin-4 and recapitulates the Syne $\%$ hearing loss phenotype in mice. We show that the cellular phenotype in Syne4\% mice is not correlated to the expression of Syne4, but rather to the onset of electromotility and hearing. Finally, we characterize the emergence of nesprin-4 in evolution and suggest a nesprin-4 ortholog in zebrafish.

Kinesin-1 is composed of a dimer of kinesin heavy chains and two kinesin light chains (KLCs). A recent study showed that Klc2 deficient mice exhibit hearing loss but no nuclear positioning defect in HC, which would argue against the role of kinesin-1 in nuclear positioning in cochlear 
HC (Ingham et al, 2019). However, as other KLCs exist in mammals, we suggest that Klc2 is redundant for nucleus positioning but required for another cellular function, such as phospholipid processing, as suggested by the authors.

We show that while delivery of the WT Syne4 by AAV is sufficient to prevent nucleus mislocalization, $\mathrm{OHC}$ death, and hearing loss, delivery of Syne4 in which the kinesin binding motif has been mutated (Syne $4^{A A}$ ) leads to no rescue whatsoever. Moreover, AAV.Syne $4^{\mathrm{AA}}$ recreates the Syne $4^{-/}$phenotype in Syne $4^{+}$mice. This effect can be interpreted as dominant-negative, in which nesprin- $4^{\mathrm{AA}}$ competes with endogenous nesprin-4 over the binding with SUN1, thus preventing a functional nucleus-cytoskeleton interaction.

The fact that we did not observe (both in this work and in (Taiber et al, 2020; Horn et al, 2013)) any effect on IHC nucleus position or survival, nor did the mice exhibit any overt vestibular behavior, is intriguing. Why is nesprin-4 so critical for OHC function and survival but not for IHC or VHC? Our data show that the onset of the phenotype in OHC correlates with the onset of electromotility. We show that while Syne4 is expressed as early as E16, there no difference in nucleus position until around P12-P14. Non-linear membrane capacitance, used as a proxy of electromotility, cannot be detected before P9 and continues to increase until P17-18 (Bai et al, 2019). DPOAE measurements, which are used as an in-vivo functional assessment of $\mathrm{OHC}$ function, are first identified at P11 for $8 \mathrm{kHz}$ and $\mathrm{P} 13$ for $30 \mathrm{kHz}$, with growing amplitudes over the next two weeks (Narui et al, 2009). Together, these observations suggest that at the time of phenotype onset in Syne4\% mice, the organ of Corti should be subjected to vertical pulling and pushing forces associated with $\mathrm{OHC}$ electromotility (Figure 7). These observations therefore support the hypothesis that nesprin-4/kinesin-1 nuclear positioning mechanism is necessary for holding the nucleus in place at the onset of somatic electromotility that is restricted to OHCs.

It is interesting to note that IHC and VHC express other nesprins as well, such as nesprin 1 and nesprin 2. These nesprins are expressed at lower levels in IHC and VHC but like for the case of nesprin-4 their expression patterns are similar to that of OHCs (Orvis et al, 2021). This observation suggests that perhaps low levels of other nesprins are sufficient to position the nucleus in $\mathrm{OHCs}$ in early stages but are not sufficient to hold it once electromotility starts. 


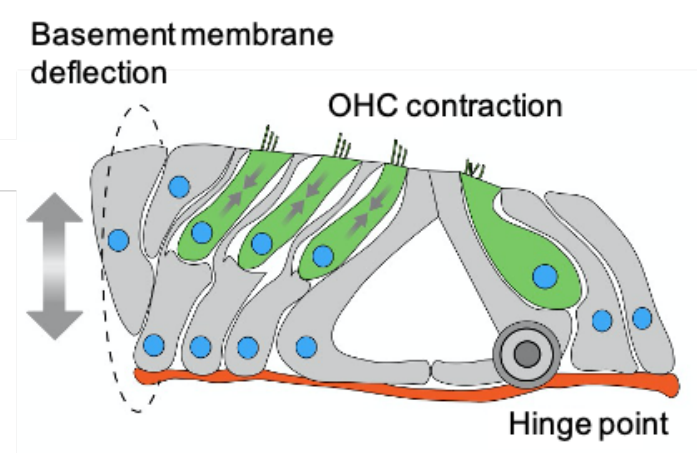

Figure 7. Schematic model depicting mechanical forces applied on OHC nuclei of mature organ of Corti.

Our results are consistent with a kinesin-1 dependent cargo model of nucleus positioning in cochlear OHC, as was suggested for myotubes (Wilson \& Holzbaur, 2012). While other mechanisms of nucleus positioning were demonstrated previously in other cell types, such as anti-parallel sliding microtubules in myotubes (Metzger et al, 2012) and dynein-mediated transport In the developing eye of drosophila (Mosley-Bishop et al, 1999), these are likely not sufficient for adult OHC given the results presented here.

It remains unclear why nuclear mislocalization leads to $\mathrm{OHC}$ death. The changes in subcellular organization we observe could impair cellular function and damage organelles. In addition, mechanical strain on nuclei has been shown to lead to NE rupture and DNA damage, with LINC complex proteins implicated in several cell types (Wong \& Stewart, 2020). Furthermore, the LINC complex can regulate gene expression via interaction with chromatin (Uzer et al, 2018). Intriguingly, our TEM data did not reveal any clear change in the structure of the NE or in the organization of the chromatin, yet autophagosomes and subcisternae membrane defects were detected in the cytoplasm. This suggests that nuclear mislocalization causes cellular damage and stress independent of damage inflicted on the nucleus itself. Further investigation of these aspects could elucidate important roles of the interplay between mechanical forces and organelle positioning in maintaining cellular homeostasis.

While IHC are very well conserved, appearing in all vertebrates, OHC, with their distinct behavior of electromotility, are unique to mammals (Manley et al, 2004). Many key OHC genes can be found in close relatives of mammals in the vertebrate lineage, with evidence showing the 
adaptation they underwent to enable the emergence of this highly specialized cell. A classic example of this principle is prestin, the motor of electromotility and a canonical OHC marker, that is ubiquitous in chordates (Okoruwa et al, 2008). VHC express prestin but do not exhibit electromotility (Adler et al, 2003). Spectrin beta-V, which is part of the lateral wall cytoskeletal complex, has also undergone evolutionary changes to support the emergence of electromotility (Cortese et al, 2017).

Alignment of human nesprin proteins against the Branchiostoma floridae genome showed that nesprin-4 is unique to vertebrates, while nesprin-1 and nesprin-2 are not. This could mean that nesprin-4 evolved due to a truncation event in one of the ancestral nesprin proteins. Our evolutionary analysis showed that "uncharacterized protein LOC777613" in the zebrafish genome clusters with mammalian nesprin-4. Examination of the expression of this gene in published datasets reveals that it is probably upregulated in hair cells. Xenopus tropicalis has nesprin-4 as well, but no expression or localization data are available at this point. The indirect evidence provided by our evolutionary analysis suggests that the emergence of nesprin-4 in evolution was one of many events enabling the subsequent development of the specialized mammalian OHC. As zebrafish hair cells are not electromotile, investigating the role of the putative zebrafish nesprin-4 could put to test our hypothesis regarding the role of electromotility in this phenotype.

\section{Materials and methods}

\section{Mice}

All animal procedures were approved by the Animal Care and Use Committee (IACUC) at Tel Aviv University (01-17-101) and performed according to the NIH Guide for the Care and Use of Laboratory Animals. For every experiment, the age is specified in the figure legend. Mice were maintained on a C57BI/6J background. Genotyping was performed on DNA prepared from ear punch biopsies, and extracted and amplified using the KAPA HotStartMouse Genotyping Kit (Sigma, KK7352). Genotyping primers for the WT allele were WT_FWD (5ACTCCCAGCTCCAAGCTACA-3) and WT_REV (5-GCAGAGCCAAAGAAACCAAG-3), and for the galactosidase gene were LacZ_FWD (5-GTCTCGTTGCTGCATAAACC-3) and LacZ_REV (5- 
TCGTCTGCTCATCCATGACC-3). Cycling conditions were an initial 3-min denaturation at $95^{\circ} \mathrm{C}$ followed by 35 cycles of $30 \mathrm{~s} 95^{\circ} \mathrm{C}, 30 \mathrm{~s} 60^{\circ} \mathrm{C}$, and $30 \mathrm{~s}$ at $72^{\circ} \mathrm{C}$, with a final elongation of $3 \mathrm{~min}$ at $72^{\circ} \mathrm{C}$. PCR products were loaded into a $2 \%$ agarose ethidium-bromide gel for electrophoresis.

\section{Plasmids and AAV production}

pEMTB-3xGFP was a gift from Eran Perlson. The AAV2 plasmid containing the Syne4 coding sequence was previously generated (Taiber et al, 2021). To introduce the desired WD to AA substitution, a gene block was synthetized, and restriction ligation cloning was performed. AAV.Syne ${ }^{\text {WD }}$ viral preps were generated as described before (Taiber et al, 2021). AAV.Syne ${ }^{\mathrm{AA}}$ was produced at Tel Aviv University using AAVpro Purification Kit Maxi (Takara) according to the manufacturer's instructions. Briefly, $1215 \mathrm{~cm}$ plates of HEK293T cells were triple-transfected with the AAV9-PHP.B cap plasmid, pAd5 adenovirus helper plasmid, and insert (ITR) Syne4 ${ }^{\text {AA }}$ plasmid. $72 \mathrm{~h}$ after transfection cells were harvested and viral vectors extracted. Viral titers were calculated based on qPCR amplification with the following primers: Syne4_FWD (5cctcttcccatgagcatcaa-3), Syne4_REV (5- ccggaagttcaacctcaaca-3). The AAV2/9.PHP.B.CMV.3xFLAG.Syne4 ${ }^{W D} . \mathrm{bGH}$ titer was $7.7 \mathrm{E}+12 \mathrm{gc} / \mathrm{ml}$, and the AAV2/9.PHP.B.CMV.3xFLAG.Syne4 ${ }^{\mathrm{AA}}$.bGH titer was $1.5737 \mathrm{E}+13 \mathrm{gc} / \mathrm{ml}$. Vectors were aliquoted into $10 \mu \mathrm{l}$ vials and stored at $-80^{\circ} \mathrm{C}$ until use.

\section{Animal surgery}

Animal surgery was performed as described previously (Taiber et al, 2020). Briefly, A posteriorsemicircular canal (PSCC) injection was carried out in mice at P0-P1.5. Mice were anesthetized by induced hypothermia and kept on a cold surface throughout the procedure. Vector solution (1.0-1.2 ul) was aspirated into a borosilicate glass pipette held by a stereotaxic device and connected to a CMA 102 Microdialysis Pump (CMA, Sweden). Once identified, the PSCC was gently punctured and the virus was microinjected for $\sim 2 \min (\sim 10 \mathrm{nl} / \mathrm{s})$. After surgery, mice were placed on a heating pad for recovery before being returned to their mothers. 


\section{Auditory testing}

$A B R$ and DPOAE, measurements were performed as described previously (Taiber et al, 2020). Briefly, mice anesthetized by intra-peritoneal injection of a combination of ketamine $(100 \mathrm{mg} / \mathrm{kg})$ and xylazine $(10 \mathrm{mg} / \mathrm{kg})$. Mice were presented with click stimuli and pure tones at $6,12,18,24$, 30 , and $35 \mathrm{khz}$, at intensities ranging from 10 to $90 \mathrm{~dB}-\mathrm{SPL}$, in steps of $5 \mathrm{~dB}$. All measurements were performed using an RZ6 multiprocessor, MF1 speakers (Tucker-Davis Technologies, Alachua, FL), and an ER-10b+ microphone (Etymotic Research, Elk Grove Village, IL), and analyzed using BioSigRZ software (Tucker-Davis Technologies, Alachua, FL) and a designated R algorithm (Rstudio, Boston, MA). All experiments were performed by the same tester.

\section{Cell culture}

HEK293 and CHO cells were cultured in Dulbecco's Modified Eagle's Medium supplemented with 10\% FBS, 1\% penicillin, and 1\% L-glutamine (Biological Industries) in a humidified incubator at $37^{\circ} \mathrm{C}$ with $5 \% \mathrm{CO}_{2}$. HEK293 cells were transfected using homemade $\mathrm{PEI}$ and $\mathrm{CHO}$ cells were transfected using LT-1 according to the manufacturer's instructions. For protein localization experiments, $\mathrm{CHO}$ cells were co-transfected with either nesprin-4 ${ }^{\mathrm{WT}}$ or nesprin-4 ${ }^{\mathrm{AA}}$ plasmids and pEMTB-3xGFP to visualize the cytoplasm.

\section{Immunofluorescence}

Inner ear dissection and staining were performed as previously described (Taiber et al, 2020). Samples were imaged using a Zeiss LSM. 880 (Zeiss, Oberkochen, Germany). CHO cells were fixed in 5\% PFA for $30 \mathrm{~min}$, permeabilized in $0.2 \%$ triton for $10 \mathrm{~min}$, and blocked in 5\% BSA for $1 \mathrm{~h}$. Antibodies were diluted in 5\% BSA for cells and in primary antibody diluent (BarNaor) for inner ear samples. Antibody staining concentrations were as follows: rabbit polyclonal myosin VIla (Proteus Biosciences, 25-6790) 1:250, rabbit anti-FLAG (Abcam, ab205606) 1:100, DAPI (Abcam ab228549) 1:1,000, and goat anti-rabbit Alexa Fluor 488 (Cell Signaling 4412s) 1:250. 


\section{Image analysis}

All data processing was performed off-line using commercial software packages (MATLAB R2019b, MathWorks Inc, Natick, MA,and Fiji). For 3D surface projections, Imaris 8.4 software was used (Bitplane, Belfast, UK). Nuclei position was extracted using a semi-automatic custom-made code in Matlab. For each cell, the positions of the apical surface, nucleus centroid and basal end of the cell were manually marked as reference points. Then, a smooth curve delineating the main axis of the cell was fitted using the marked reference points. The total length of the cell was estimated by calculating the total length of the fitted curve. The nucleus position with respect to the apical surface was estimated as the length of the curve between the apical and nuclear reference points. Nuclear recruitment was quantified in custom-made Fiji macro. Nuclei were segmented using the DAPI channel and auto-thresholding. The entire cell was segmented using the microtubules channel. Cytoplasmic intensity was measured by measuring the intensity in the segment of the entire cell excluding the segment nucleus. Perinuclear intensity was measured in $1.1 \mu \mathrm{m}$ band surrounding the nucleus.

\section{Transmission electron-microscopy}

Following decapitation and extraction of whole inner ears, $1 \mathrm{ml}$ of fixation solution $(2.5 \%$ gluteraldehyde, 4\% PFA, $0.1 \mathrm{M}$ sodium cacodylate, $5 \mathrm{mM} \mathrm{CaCl}$, and $2 \mathrm{mM} \mathrm{MgCl}$ ) was slowly injected through the round window and samples were transferred to a $10 \mathrm{ml}$ tube with fixative for $2 \mathrm{~h}$ at RT. After dissection samples were incubated in $1 \%$ osmium tetroxide with $1.2 \%$ potassium ferricyanide for $40 \mathrm{~min}$. Samples were then washed 3 times, stained with $1 \%$ uranyl acetate for $1 \mathrm{~h}$, dehydrated in graded acetone dilutions, embedded with epon resin and polymerized for 2 days at $60^{\circ} \mathrm{C}$. Sixty $\mathrm{nm}$ sections were cut (Leica UC7 ultramicrotome), transferred to copper grids and stained with UranyLess (EMS). Samples were imaged using Zeiss Libra TEM at $80 \mathrm{kV}$.

\section{Western blot and immunoprecipitation}

HEK293 cells were cultured in Dulbecco's Modified Eagle's Medium supplemented with 10\% FBS, $1 \%$ penicillin, and 1\% L-glutamine (Biological Industries), transfected using jetPEI (Polyplus) 
according to manufacturer's instructions and harvested $48 \mathrm{~h}$ after transfection. One $10 \mathrm{~cm}$ plate per condition was lysed $1 \mathrm{ml}$ RIPA buffer (Sigma) and Halt Protease Inhibitor Cocktail (Thermo). Cells were placed on an end-over-end shaker for $1 \mathrm{~h}$ at $4^{\circ} \mathrm{C}$ followed by centrifugation at 16,000 $\mathrm{g}$ for $15 \mathrm{~min}$ at $4^{\circ} \mathrm{C}$. $20 \mathrm{ul}$ of the resulting supernatant was used as input and the rest was incubated with EZview anti-FLAG M2 beads (F2426, Sigma) for 1-2 $\mathrm{h}$ at $4{ }^{\circ} \mathrm{C}$ on an end-over-end shaker. Beads were then washed and eluted in sample buffer according to manufacturer's instructions and loaded into a 10\% SDS-PAGE gel. Samples were then transferred onto a nitrocellulose membrane which was then blocked in 3\% skimmed milk (BD Difco). Membranes were then subjected to immunoblotting. Kif5b was detected by anti Kif5b (Abcam, ab167429). FLAG was detected using rabbit anti DDDDK antibody (Abcam, ab205606). Blots were visualized using anti rabbit HRP antibody (Cell Signaling Technologies, 7074) and SuperSignal West Pico PLUS Chemiluminescence Substrate (Thermo Scientific).

\section{Evolutionary analysis}

The nesprin protein sequences were aligned using MUSCLE (Edgar, 2004). The maximumlikelihood phylogeny was generated using IQ-Tree (Nguyen et al, 2015) with the LG+F+R5 model which was the best fitting model both according to the Bayesian information criterion (BIC) and corrected Akaike information criterion. Support values of the phylogenetic tree are derived from 1,000 ultrafast bootstrap replicates (Minh et al, 2013).

\section{In situ hybridization}

Single molecule fluorescent in situ hybridization ( $\mathrm{smFISH}$ ) detection of expression of Syne4 was performed as described previously (Kolla et al, 2020). Briefly, cochleae were dissected and fixed in $4 \%$ PFA overnight at $4^{\circ} \mathrm{C}$. Postnatal day 11 cochleae were decalcified in $0.25 \mathrm{M}$ EDTA overnight. Then, Samples were washed, subjected to a sucrose gradient and embedded in OCT. Next, samples were sectioned on a cryostat at $10 \mu \mathrm{m}$ thickness. Probes for Syne4, Pvalb, a marker of hair cells, and the RNAscope Fluorescent Multiplex Reagent Kit were purchased from Advanced Cell Diagnostics. Hybridization was performed following the manufacturer's suggested protocol. Results were imaged using a Zeiss LSM 710 confocal microscope. 


\section{Statistics}

Statistical tests, group sizes, and $\mathrm{P}$ values are noted in the figure legends. Littermates were randomized to the different experiment groups. No blinding was performed, and all tests were carried out by the same tester. Objective measures were preferred when possible. These include hair cell counts, DPOAE thresholds, image analysis, and evolutionary analyses. Statistical analyses were performed using Prism 8 software (GraphPad, San Diego, CA). When required, Shapiro-Wilk and Kolmogorov-Smirnov were used to test data for normality. For comparisons of more than two groups or conditions, the Tukey post hoc test was used to adjust $P$ values when data passed normality tests and Kruskal-Wallis test with Dunn's correction was used when data did not pass normality tests.

\section{Data availability}

This study includes no data deposited in external repositories. All data and/or codes are available upon request.

\section{Acknowledgements}

The authors wish to thank Daniel Nataf, Adi Barzel, and Michal Lisnyansky Bar-El for technical help and advice. The research was funded by the National Institutes of Health/NIDCD R01DC011835 (K.B.A.) and DC000059 (M.W.K.), the United States-Israel Binational Science Foundation (BSF) 01027150, Jerusalem, Israel (K.B.A.), the Israel Science Foundation (grant no. 1763/20) (K.B.A., M.W.K.), and the European Research Council (ERC) under the European Union's Horizon 2020 Research and Innovation Program, Grant Agreement No. 682161 (D.S.). K.B.A. is an incumbent of the Drs. Sarah and Felix Dumont Chair for Research of Hearing Disorders. U.M. is supported by the Chan-Zuckerberg Initiative Imaging Scientist Award, NSF NeuroNex Award No. 2014862, and the Hillblom Foundation. U.M. and L.R.A. are supported by National Institutes of Health (NIH) grant no. R21 DC018237, the Waitt Foundation, the Grohne Foundation, and NIH-NCI CCSG: P30 014195. This work was performed in partial fulfillment of the requirements for a Ph.D. degree by Shahar Taiber, recipient of the Klass Family Fellowship, at the Faculty of Medicine, Tel Aviv University, Israel. 


\section{Author contributions}

ST, KBA, and DS designed the study and interpreted the results. ST performed molecular biology experiments, mouse injections, auditory testing, cochlear dissections, and immunofluorescence, and analyzed the data. LA and UM performed electron microscopy and analyzed the results. RH and MWK designed and performed RNAscope experiments. OG and RC performed cell culture experiments and microscopy. YM performed evolutionary analyses. RC wrote MATLAB codes for image analysis. ST, KBA, and DS wrote the manuscript. All authors contributed to the article and approved the submitted version.

\section{Conflict of interest}

The authors declare no conflict of interests.

\section{References}

Adler HJ, Belyantseva IA, Merritt RC, Frolenkov GI, Dougherty GW \& Kachar B (2003) Expression of prestin, a membrane motor protein, in the mammalian auditory and vestibular periphery. Hear. Res. 184: 27-40

Ashmore J (2019) Outer hair cells and electromotility. Cold Spring Harb. Perspect. Med. 9:

Bai JP, Navaratnam D \& Santos-Sacchi J (2019) Prestin kinetics and corresponding frequency dependence augment during early development of the outer hair cell within the mouse organ of Corti. Sci. Rep. 9: 16460

Cortese M, Papal S, Pisciottano F, Elgoyhen AB, Hardelin J-P, Petit C, Franchini LF \& El-Amraoui A (2017) Spectrin $\beta V$ adaptive mutations and changes in subcellular location correlate with emergence of hair cell electromotility in mammalians. Proc. Natl. Acad. Sci. USA 114: 2054-2059

Crisp M, Liu Q, Roux K, Rattner JB, Shanahan C, Burke B, Stahl PD \& Hodzic D (2006) Coupling of the nucleus and cytoplasm: Role of the LINC complex. J. Cell Biol. 172: 41-53

Edgar RC (2004) MUSCLE: Multiple sequence alignment with high accuracy and high 
throughput. Nucleic Acids Res. 32: 1792-1797

Grady RM, Starr DA, Ackerman GL, Sanes JR \& Han M (2005) Syne proteins anchor muscle nuclei at the neuromuscular junction. Proc. Natl. Acad. Sci. USA 102: 4359-4364

Haque F, Lloyd DJ, Smallwood DT, Dent CL, Shanahan CM, Fry AM, Trembath RC \& Shackleton S (2006) SUN1 interacts with nuclear lamin A and cytoplasmic nesprins to provide a physical connection between the nuclear lamina and the cytoskeleton. Mol. Cell. Biol. 26: 37383751

Horn HF, Brownstein Z, Lenz DR, Shivatzki S, Dror AA, Dagan-Rosenfeld O, Friedman LM, Roux KJ, Kozlov S, Jeang KT, Frydman M, Burke B, Stewart CL \& Avraham KB (2013) The LINC complex is essential for hearing. J. Clin. Invest. 123: 740-750

Ingham NJ, Pearson SA, Vancollie VE, Rook V, Lewis MA, Chen J, Buniello A, Martelletti E, Preite L, Lam CC, Weiss FD, Powis Z, Suwannarat P, Lelliott CJ, Dawson SJ, White JK \& Steel KP (2019) Mouse screen reveals multiple new genes underlying mouse and human hearing loss. PLoS Biol. 17: e3000194

Kolla L, Kelly MC, Mann ZF, Anaya-Rocha A, Ellis K, Lemons A, Palermo AT, So KS, Mays JC, Orvis J, Burns JC, Hertzano R, Driver EC \& Kelley MW (2020) Characterization of the development of the mouse cochlear epithelium at the single cell level. Nat. Commun. 11: 2389.

Manley GA, Fay RR \& Popper AN eds. (2004) Evolution of the vertebrate auditory system New York, NY: Springer New York

Matern MS, Beirl A, Ogawa Y, Song Y, Paladugu N, Kindt KS \& Hertzano R (2018) Transcriptomic profiling of zebrafish hair cells using RiboTag. Front. Cell Dev. Biol. 6: 47

Mellado Lagarde MM, Drexl M, Lukashkin ANN, Zuo J \& Russell IJ (2008) Prestin's role in cochlear frequency tuning and transmission of mechanical responses to neural excitation. Curr. Biol. 18: 200-202

Metzger T, Gache V, Xu M, Cadot B, Folker ES, Richardson BE, Gomes ER \& Baylies MK (2012) MAP and kinesin-dependent nuclear positioning is required for skeletal muscle function. Nature 484: 120-124

Minh BQ, Nguyen MAT \& Von Haeseler A (2013) Ultrafast approximation for phylogenetic bootstrap. Mol. Biol. Evol. 30: 1188-1195 
Morimoto A, Shibuya H, Zhu X, Kim J, Ishiguro KI, Han M \& Watanabe Y (2012) A conserved KASH domain protein associates with telomeres, SUN1, and dynactin during mammalian meiosis. J. Cell Biol. 198: 165-172

Mosley-Bishop KL, Li Q, Patterson K \& Fischer JA (1999) Molecular analysis of the klarsicht gene and its role in nuclear migration within differentiating cells of the Drosophila eye. Curr. Biol. 9: 1211-1220

Narui Y, Minekawa A, lizuka T, Furukawa M, Kusunoki T, Koike T \& Ikeda K (2009) Development of distortion product otoacoustic emissions in C57BL/6J mice. Int. J. Audiol. 48: 576-581

Nguyen LT, Schmidt HA, Von Haeseler A \& Minh BQ (2015) IQ-TREE: A fast and effective stochastic algorithm for estimating maximum-likelihood phylogenies. Mol. Biol. Evol. 32: $268-274$

Okoruwa OE, Weston MD, Sanjeevi DC, Millemon AR, Fritzsch B, Hallworth R \& Beisel KW (2008) Evolutionary insights into the unique electromotility motor of mammalian outer hair cells. Evol. Dev. 10: 300-315

Orvis J, Gottfried B, Kancherla J, Adkins RS, Song Y, Dror AA, Olley D, Rose K, Chrysostomou E, Kelly MC, Milon B, Matern MS, Azaiez H, Herb B, Colantuoni C, Carter RL, Ament SA, Kelley MW, White O, Bravo HC, et al (2021) gEAR: Gene Expression Analysis Resource portal for community-driven, multi-omic data exploration. Nat. Methods 18: 843-844

Padmakumar VC, Libotte T, Lu W, Zaim H, Abraham S, Noegel AA, Gotzmann J, Foisner R \& Karakesisoglou I (2005) The inner nuclear membrane protein Sun1 mediates the anchorage of Nesprin-2 to the nuclear envelope. J. Cell Sci. 118: 3419-3430

Raphael Y \& Altschuler RA (2003) Structure and innervation of the cochlea. Brain Res. Bull. 60: $397-422$

Roux KJ, Crisp ML, Liu Q, Kim D, Kozlov S, Stewart CL \& Burke B (2009) Nesprin 4 is an outer nuclear membrane protein that can induce kinesin-mediated cell polarization. Proc. Natl. Acad. Sci. USA 106: 2194-2199

Steiner AB, Kim T, Cabot V \& Hudspeth AJ (2014) Dynamic gene expression by putative hair-cell progenitors during regeneration in the zebrafish lateral line. Proc. Natl. Acad. Sci. USA

111: E1393-401 
Taiber S, Cohen R, Yizhar-Barnea O, Sprinzak D, Holt JR \& Avraham KB (2020) Neonatal AAV gene therapy rescues hearing in a mouse model of SYNE4 deafness. EMBO Mol. Med. 13: e13259

Uzer G, Bas G, Sen B, Xie Z, Birks S, Olcum M, McGrath C, Styner M \& Rubin J (2018) Sunmediated mechanical LINC between nucleus and cytoskeleton regulates $\beta$ catenin nuclear access. J. Biomech. 74: 32-40

Wilhelmsen K, Litjens SHM, Kuikman I, Tshimbalanga N, Janssen H, Van Bout I Den, Raymond K \& Sonnenberg A (2005) Nesprin-3, a novel outer nuclear membrane protein, associates with the cytoskeletal linker protein plectin. J. Cell Biol. 171: 799-810

Wilson MH \& Holzbaur ELF (2012) Opposing microtubule motors drive robust nuclear dynamics in developing muscle cells. J. Cell Sci. 125: 4158-4169

Wilson MH \& Holzbaur ELF (2015) Nesprins anchor kinesin-1 motors to the nucleus to drive nuclear distribution in muscle cells. Dev. 142: 218-228

Wong X \& Stewart CL (2020) The laminopathies and the insights they provide into the structural and functional organization of the nucleus. Annu. Rev. Genomics Hum. Genet. 21: 263-288 